\title{
Segmentation of Multimodal MRI of Hippocampus Using 3D Grey-Level Morphology Combined with Artificial Neural Networks
}

\author{
Roger Hult ${ }^{1}$ and Ingrid Agartz ${ }^{2}$ \\ 1 Centre for Image Analysis, Uppsala University, \\ Lägerhyddsvägen 3, SE-752 37 Uppsala, Sweden \\ rogerh@cb.uu.se \\ 2 Dept. Clinical Neuroscience, Human Brain Informatics, \\ Karolinska Institutet, SE-171 75, Stockholm, Sweden
}

\begin{abstract}
This paper presents an algorithm for improving the segmentation from a semi-automatic artificial neural network (ANN) hippocampus segmentation of co-registered T1-weigthted and T2-weighted MRI data, in which the semi-automatic part is the selection of a boundingbox. Due to the morphological complexity of the hippocampus and the difficulty of separating from adjacent structures, reproducible segmentation using MR imaging is complicated.

The grey-level thresholding uses a histogram-based method to find robust thresholds. The T1-weighted data is grey-level eroded and dilated to reduce leaking from hippocampal tissue to the surrounding tissues and selecting possible foreground tissue. The method is a $3 \mathrm{D}$ approach, it uses $3 \times 3 \times 3$ structure element for the grey-level morphology operations and the algorithms are applied in the three directions, sagittal, axial, and coronal, and the results are then combined together.
\end{abstract}

\section{Introduction}

The hippocampus is a structure that is located in the medial temporal lobe of the human brain and is considered to be a part of the limbic system: it is divided into three parts: head, body, tail. The volume of the hippocampus is important in the studies in schizophrenia research. See Fig. 1 for a 3D view of the shape of the two hippocampi and a segmented sagittal slice.

There are several other techniques, which segment the hippocampus described in the literature. There are methods which depend on active shape models (ASM) or point distribution models (PDM) [1], the theory behind those kind of models is described in [2], [3]. The shape of the region that is segmented is changed iteratively until a fit is found. A good startposition is important for this segmentation to work. Regional fluid registration has also been used for segmentation of the hippocampus [4]. There are examples of matching the hippocampus to a brain atlas [5], [6].

Three MR modalities are used for the segmentation of the hippocampus, T1weighted, T2-weighted, and a continously classified stereo image. See Fig. 2 for a 


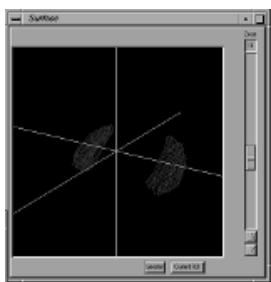

(a)

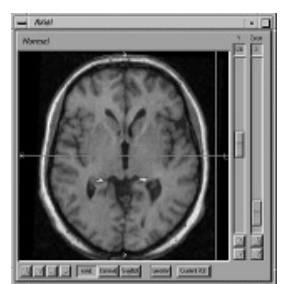

(b)

Fig. 1. Segmented hippocampi viewed as contours. a) Contours in 3D. b) Contours in the axial plane

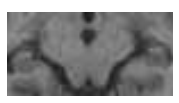

(a)

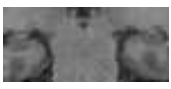

(b)

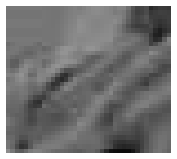

(c)

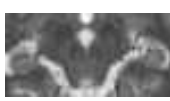

(d)

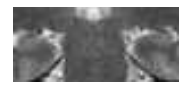

(e)

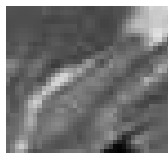

(f)

Fig. 2. Slices of the different modalities in the bounding box.: a) A T1-weighted axial slice. b) A T1-weighted coronal slice. c) A T1-weighted sagittal slice. d) A T2-weighted axial slice. e) A T2-weighted coronal slice. f) A T2-weighted sagittalslice

view of slices in the two different modalities used in the segmentation algorithm in this paper. Only what is within in the bounding box is showed. The classified image is used by the ANN method in BRAINS2, the two other modalities are used by grey-level morphology segmentation. Hippocampus is, for several reasons, a difficult region to delineate automatically. Since it is a bent structure, the cross-sections of the hippocampus change considerably in all three planes, and some parts of the hippocampus are very difficult to separate from surrounding brain structures on MR images. The hippocampus is easy to segment on the most lateral slice in the sagittal plane. On more medial parts of the hippocampus, the head and tail are seen as two independent structures. On proceeding further from the medial parts, the hippocampus becomes progressively harder to delineate.

Before the segmentation algorithm described herein can be applied, the hippocampus must be pre-segmented using an ANN approach in the program BRAINS2 [7] from the Mental Health Clinical Research Center, The University of Iowa College of Medicine and Hospitals and Clinics, Iowa City USA. This segmentation involves registration [8] of the T1-weighted and T2-weighted images and aligning them in the Talairach atlas space with the brain realigned in the AC-PC line. The AC-PC-plane is a line from one structure in the brain, the anterior commissure, to another part of the brain, the posterior commissure. The brain is then resized to fit an orthogonal frame based around the origin. The resizing is done using a 6-point linear transformation, and these methods therefore have a limited capability of correcting for any variations other than size. Anatomical localisation of an area of interest is then achieved by referring its 
stereotactic co-ordinates in an atlas textbook, e.g. the Talairach and Tournoux atlas [9].

When the T1-weighted image has been set in the Talairach atlas space, and the T2-weighted images has been registered to the realigned T1-weighted image, the images are classified using a multimodal discriminant classifier. This classification recudes the variability in signal intensity across individual images sets [10]. After realignment the data is reduced to 8-bit data, wich allowes for only 256 grey-levels. After this classification there are specific intervals for the different kinds of tissue in the brain. These values are: cerebral spinal fluid (CSF) 10-70, grey matter (GM) 71-190, and white matter (WM) 191-250. Each voxel in the stereo image is assigned an intensity value based on the weights assigned by the discriminant function, reflecting the relative combinations of CSF, GM, and WM in a given voxel, producing a continuous classified image. There is also a discrete classified image: mostly white matter voxels are assigned the intensity 250, mostly grey matter voxels are assigned the intensity 130, and CSF voxels are assigned an intensity of 10 .

For the segmentation of the hippocampus artificial neural nets [11], [12] (ANN) are being used. The ANN uses a fully connected feed-forward neural net with three layers. The output node was used as a "fuzzy value" to indicate if a voxel was in the ROI or not. The search space for the ANN in the normalised space was determined by creating a mask representing all manual traces in the training set. The input vector for the ANN consists of the intensity from the current voxel and also from neighbouring voxels. A bounding box is used to limit the search and contains both the left and right hippocampus. If the box is much larger than the hippocampus the ANN will fail in the segmentation process, which may result in an extra region close to the hippocampus. When the bounding box has been selected, the neural net generates a mask.

See Fig. 3 for sagittal slices of T1-weighted and T2-weighted data and the resulting slice from the classified volume used by the ANN method to generate a mask of the hippocampus. The classified volume is used by the ANN method to generate a mask of the hippocampus. The mask generated is smaller than the hippocampus, sometimes only $60 \%$ of the volume found when compared to a manual segmentation.

The ANN was trained from hippocampi manually traced in the sagittal plane. There are separate windows for coronal, axial, sagittal, and 3D ROI views. For a detailed explanation on how this should be done, see elsewhere [13]. An experienced tracer can delineate the hippocampus in about $60-90 \mathrm{~min}$. The combined

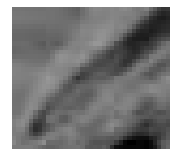

(a)

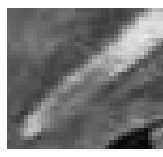

(b)

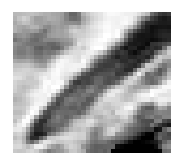

(c)

Fig. 3. Sagittal slices of the different modalities in the bounding box.: a) A T1weigthted slice. b) A T2-weighted slice. c) A classified stereo slice 
ANN and grey-level morphology approach only takes a few minutes to run on an ordinary workstation. The manual correction needed is then reduced to small corrections on a few slices only.

\section{Methods}

\section{$2.1 \quad$ Overview}

The method is based on pre-segmented data and grey-level morphology combined with anatomical knowledge and binary morphology. In addition to the original MRI-data, T1-weighted and T2-weighted, called OrgImageT1 and OrgImageT2, four volumes are calculated using grey-level morphology [14], [15], [16]. The original MRI-data is grey-level-eroded using a $3 \times 3 \times 3$ structure element; the new volumes are called T1MinImage and T2MinImage. The original MRIdata is also grey-level-dilated using a $3 \times 3 \times 3$ structure element; those volumes are called T1MaxImage and T2MaxImage. See Fig. 4 for examples how these volumes may look. From the T1MinImage the foreground is thresholded using kernel density estimates (continuous histogram (KDE)) [17], [18], [19]. From this continuous histogram the second derivate is calculated and the lowest greatest maxima is selected. Initially the lateral outermost and innermost parts of the hippocampus in the sagittal plane are found. Further on, more of the head and tail of the hippocampus are found. Then the whole hippocampus is segmented in sagittal, coronal, and axial directions using information from the T1-weighted and the T2-weighted volume combined with grey-level eroded and dilated versions of the two modalities. The grey-levels are reduced to five grey-levels using a KDE approach for each slice of the images. Then the greylevel reduced versions of two modalities are thresholded between the highest and lowest grey-level found in the pre-segmented input mask. The result from the thresholding is then ANDed together, and also combined with the possible foreground.

A sequence of binary morphology operations is then performed combined with labelling and flagging using the pre-segmented volume. See Fig. 5 for an example for the result of the segmentation algorithm. The results of the segmentation are then ANDed together. The result is then labelled and flagged using the presegmented volume in all three directions. See Fig. 6.

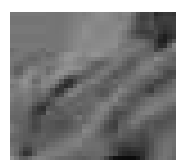

(a)

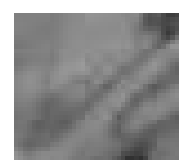

(b)

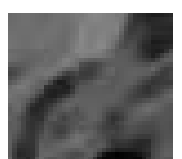

(c)

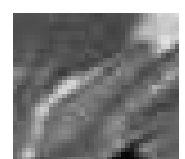

(d)

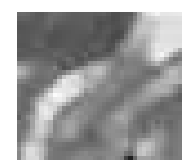

(e)

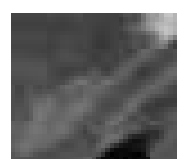

(f)

Fig. 4. Sagittal slices of the different modalities and in the bounding box.: a) A T1weighted slice. b) A T1-weighted grey-level dilated slice. c) A T1-weighted grey-level eroded slice. d) A T2-weighted slice. e) A T2-weighted grey-level dilated slice. f) A T2-weighted grey-level eroded slice 


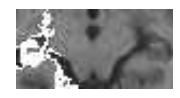

(a)

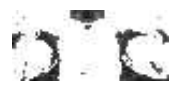

(d)

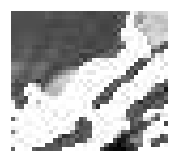

(g)

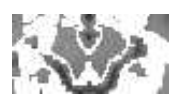

(b)

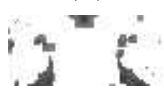

(e)

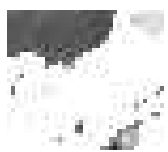

(h)

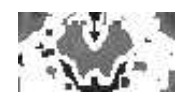

(c)

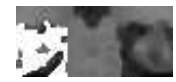

(f)

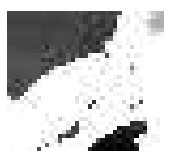

(i)

Fig. 5. Result of slices of the different modalities in the bounding box: a) A segmented axial slice. b) A segmented grey-level dilated axial slice. c) A grey-level eroded axial slice. d) A segmented coronal slice. e) A segmented grey-level dilated coronal slice. f) A grey-level eroded coronal slice. g) A segmented sagittal slice. h) A segmented grey-level dilated sagittal slice. i) A grey-level eroded sagittal slice

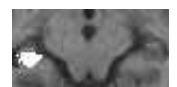

(a)

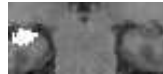

(b)

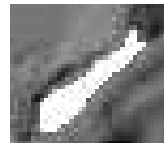

(c)

Fig. 6. Result of segmentation with $\mathrm{ANN}$ and grey-level morphology, generated by ANDing the result from Fig. 5a-i): a) A axial slice. b) A coronal slice. c) A sagital slice

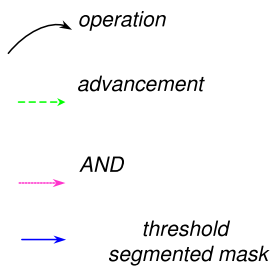

Fig. 7. Description of arrows used in the flow-charts

\subsection{Segmentation Algorithms}

The important steps of the algorithm are described using flow-charts. All binary dilations and erosions use a $3 \times 3$ structure element. All grey-level dilations and erosions use a $3 \times 3 \times 3$ structure element. Unless otherwise stated, operations on consecutive lines are performed in sequence using previous result. See Fig. 7 for a description of the arrows used in the flow-charts.

\section{Foreground detection algorithm}

The Foreground is found from T1MinImage using a KDE approach. 
input:

A grey-level eroded T1-weighted volume, T1MinImage.

output:

A mask with possible brain matter, Matter.

using the histograms

generate KDE from T1MinImage

find 2 largest local max in 2nd derivative sort

select threshold above 1 st $\max \rightarrow$ Matter

end;

\section{Lateral part of the hippocampus in sagittal slices}

The algorithm is applied from the last sagittal slice on the ANN segmented volume containing hippocampus and continues laterally until there is no more hippocampus. See Fig 8 and Fig. 9 for flow-charts of the algorithm.

input:

T1-weighted MR data of the brain, T1OrgImage.

T2-weighted MR data of the brain, T2OrgImage.

Mask with segmented hippocampus, AnnSegmMask.

Mask with possible brain matter, Matter.

output:

Mask with more lateral hippocampus, PreSegmMask.

\section{Hippocampus head and tail segmentation algorithm}

This algorithm is applied on the original data, grey-level eroded data, and greylevel dilated data; the results from those are then ANDed together. It starts at

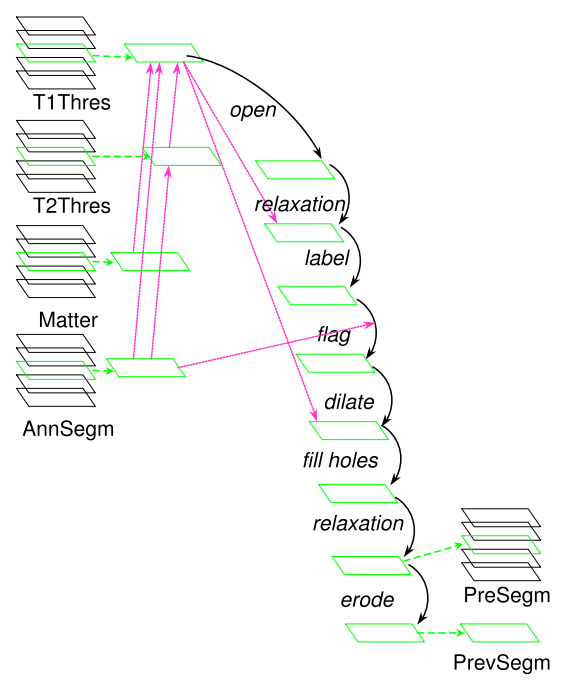

Fig. 8. Lateral part of the hippocampus in sagittal slices, start slice 


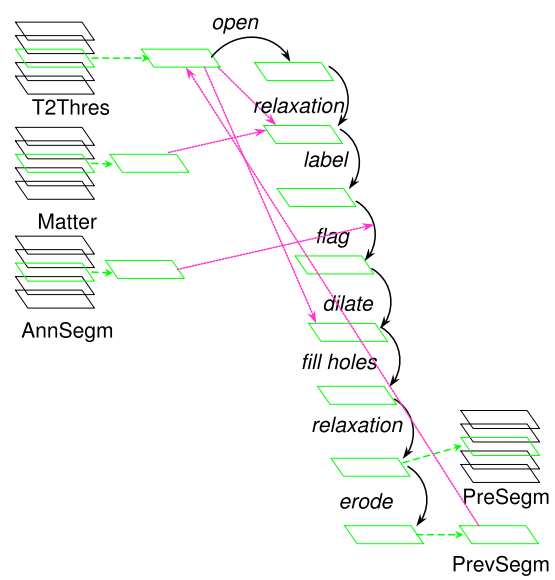

Fig. 9. Lateral part of the hippocampus in sagittal slices, traversing volume

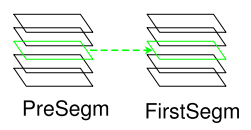

Fig. 10. Hippocampus head and tail segmentation algorithm, start slice

the last slice containing hippocampal tissue, unless that is the first or last slice within the bounding box. It is also applied in coronal and axial directions in the head and tail, and the results from the two directions and two positions are ORed together. See Fig 10 and Fig. 11 for flow-charts of the algorithm. input:

T1-weighted MR data of the brain, T1Image.

T2-weighted MR data of the brain, T2Image.

Mask with pre-segmented hippocampus, PreSegmMask.

A mask with possible brain matter, Matter.

output:

Mask with first segmented hippocampus, FirstSegmMask.

\section{Hippocampus segmentation algorithm}

This algorithm is applied on the original data, grey-level eroded data, and grey-level dilated data, and the results from those are ANDed together. It is also applied in sagittal, coronal, and axial directions, and the results from the three directions are ANDed together. See Fig 12 for a flow-chart of the algorithm.

input:

T1-weighted MR data of the brain, T1Image.

T2-weighted MR data of the brain, T2Image. 


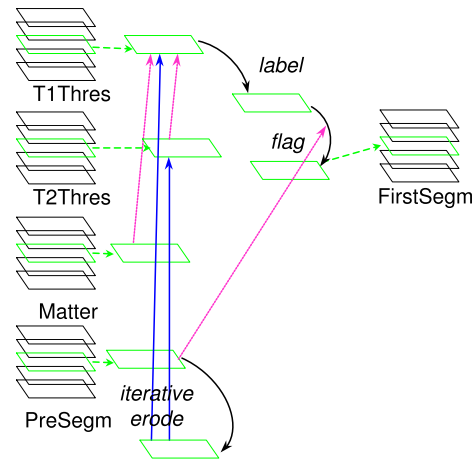

Fig. 11. Hippocampus head and tail segmentation algorithm, traversing volume

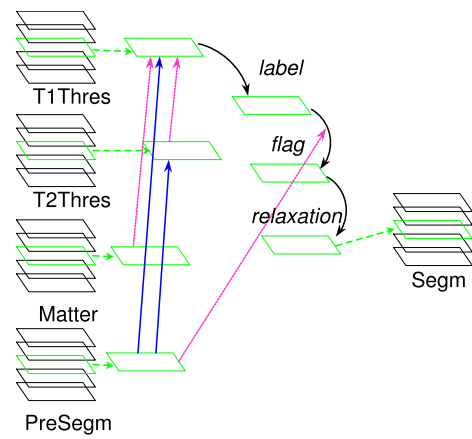

Fig. 12. Hippocampus segmentation algorithm

Mask with pre-segmented hippocampus, FirstPreSegmMask.

A mask with possible brain matter, Matter.

output:

Mask with more segmented hippocampus, SegmMask.

\section{$3 \quad$ Results}

After testing the method on thirty data sets, the method seems promising: compared to the original ANN method, its segmentation of the hippocampus is much closer to the segmentation obtained by an experienced scientist. Most often the ANN segmentation only finds about $60 \%$ of the volume compared to the result from the human tracer, whereas the combined method finds at least $90 \%$ and up to $95 \%$ of the volume found by the human tracer. The new method does not remove the human intervention completely, but minimises the manual corrections to a few slices compared to almost half the slices. 


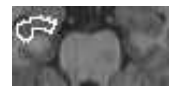

(a)

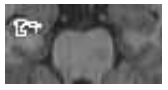

(b)

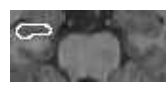

(c)

Fig. 13. Result of segmentation on an axial slice: a) Hand-traced slice. b) Slices segmented with ANN. c) Slice segmented with ANN and grey-level morphology

Since the manual drawing is only done in sagittal slices there is a risk that the consecutive slices may drift.In Fig. 13 it can be seen that the manually traced hippocampus is more jagged than the combined method. The ANN method obviously misses large areas and the combined method can expand those areas to a better segmentation of the hippocampus.

To reduce manual drawing, a 3D-approach using grey-level morphology was implemented. In this paper, earlier research on grey-level based segmentation by the first author is used [20], [21], combining an imperfect segmentation tool using ANNs with an algorithm needing some information in each slice to be segmented, in order to create a new and better algorithm.

\section{Future Work}

The development of the hippocampus segmentation will continue in order to reduce human interaction even more. There will also be further development of segmentation algorithms for other structures, using grey-level morphology and including artificial neural nets (ANN) from BRAINS2.

\section{References}

1. Kelemen, A., Szekely, G., Gerig, G.: Elastic Model-Based Segmentation of 3-D Neuroradiological Data Sets. IEEE Transactions on Medical Imaging 18 (1999) 828-839

2. Cootes, T.F., Taylor, C.J., Cooper, D.H., Graham, J.: Active Shape Models Their Training and Application. Computer Vision and Image Understanding 61 (1995) 38-59

3. Cootes, T.F., Taylor, C.J.: Active Shape Models. Draft from a forthcomming report on ASM (1998)

4. Crum, W.R., Scahill, R.I., Fox, N.C.: Automated Hippocampal Segmentation by Regional Fluid Registration of Serial MRI: Validation and Application in Alzheimer's Disease. NeuroImage 13 (2001) 847-855

5. Haller, J.W., Bannerjee, A., Christensen, G.E., Gado, M., Joshi, S., Miller, M., Sheline, M.I., Vannier, M.W., Csernansky, J.G.: Three-Dimensional Hippocampal MR Morphometry with High-Dimensional Transformation of a Neuroanatomic Atlas. Radiology 51 (1997) 993-999

6. Fischl, B., Salat, D.H., Busa, E., Albert, M., Haselgrove, M.D.C., van der Kouwe, A., Killiany, R., Kennedy, D., Klaveness, S., Montillo, A., Makris, N., Rosen, B., Dale, A.M.: Whole Brain Segmentation: Automated Labeling of Neuroanatomical Structures in the Human Brain. Neurotechnique 33 (2002) 341-355 
7. Magnotta, V.A., Harris, G., Andreasen, N.C., O'Leary, D.S., Yuh, W.T., Heckel, D.: Structural MR Image Processing using the BRAINS2 Toolbox. Computerized Medical Imaging, Graphics 26 (2002) 251-264

8. Woods, R.P., Grafton, S.T., Watson, J.D., Sicotte, N.L., Mazziota, J.C.: Improved Methods for Image Registration. Journal of Computer Assisted Tomography 22 (1998) 153-165

9. Talairach, J., Tournoux, P.: Co-planar Stereotactic Atlas of the Human Brain: 3-dimensional Proportional System-an Approach to Cerebral Imaging. Thieme Verlag, Stuttgart/New York (1988)

10. Harris, G., Andreasen, N.C., Cizadlo, T., Bailey, J.M., Bockholt, H.J., Magnotta, V.A., Arndt, S.: Improving Tissue Classification in MRI: A Three-Dimensional Multispectral Discriminant Analysis Method with Automated Training Class Selection. Journal of Computer Assisted Tomography 23 (1999) 144-154

11. Magnotta, V.A., Heckel, D., Andreasen, N.C., Cizadlo, T., Corson, P.W., Ehrhardt, J.C., Yuh, W.T.: Measurement of Brain Structures with Artificial Neural Networks: Two- and Three-Dimensional Applications. Radiology 211 (1999) 781-790

12. Pierson, R., Corson, P.W., Sears, L.L., Alicata, D., Magnotta, V., O'Leary, D.S., Andreasen, N.C.: Structural MR Image Processing Using the BRAINS2 Toolbox. NeuroImage 17 (2002) 61-76

13. Pantel, J., O'Leary, D.S., Cretsinger, K., Bockholt, H.J., Keefe, H., Magnotta, V.A., Andreasen, N.C.: A New Method for the In Vivo Volumetric Measurement of the Human Hippocampus with High Neuroanatomical Accuracy. Hippocampus 10 (2000) 752-758

14. Serra, J.: Image Analysis and Mathematical Morphology. Volume 1. Academic Press (1982)

15. Serra, J., ed.: Image Analysis and Mathematical Morphology Volume 2: Theoretical Advances. Volume 2. Academic Press (1988)

16. Gonzalez, R.C., Woods, R.E.: 8. In: Digital Image Processing. 2 edn. AddisonWesley Publishing Company, Inc. (2002) 550-560

17. Lindblad, J.: Histogram Thresholding using Kernel Density Estimates. In: Proceedings of SSAB (Swedish Society for Automated Image Analysis) Symposium on Image Analysis, Halmstad, Sweden. (2000) 41-44

18. Rosenblatt, M.: Remarks on Some Nonparametric Estimates of a Density Function. Annals of Mathematical Statistics (1956) 642-669

19. Parzen, E.: On Estimation of Probability and Mode. Annals of Mathematical Statistics 33 (1962) 1065-1076

20. Hult, R., Bengtsson, E., Thurfjell, L.: Segmentation of the Brain in MRI Using Grey Level Morphology and Propagation of Information. In: Proceedings of $11^{\text {th }}$ Scandinavian Conference on Image Analysis, Kangerlussuaq, Greenland. Volume I. (1999) 367-373

21. Hult, R.: Grey-level Morphology Based Segmentation of MRI of the Human Cortex. In: Proceedings of ICIAP01 11th International Conference on Image Analysis and Processing, Palermo, Italy. (2001) 578-583 